\title{
Some Variational Principles for Coupled Thermoelasticity
}

\author{
Francesco Marotti de Sciarra \\ Dipartimento di Ingegneria Strutturale, Università di Napoli Federico II, Via Claudio 21, 80125 Napoli, Italy
}

Correspondence should be addressed to Francesco Marotti de Sciarra; marotti@unina.it

Received 22 September 2012; Accepted 18 December 2012

Academic Editor: Oronzio Manca

Copyright (c) 2013 Francesco Marotti de Sciarra. This is an open access article distributed under the Creative Commons Attribution License, which permits unrestricted use, distribution, and reproduction in any medium, provided the original work is properly cited.

\begin{abstract}
The nonlinear thermoelasticity of type II proposed by Green and Naghdi is considered. The thermoelastic structural model is formulated in a quasistatic range, and the related thermoelastic variational formulation in the complete set of state variables is recovered. Hence a consistent framework to derive all the variational formulations with different combinations of the state variables is provided, and a family of mixed variational formulations, with different combinations of state variables, is provided starting from the general variational formulation. A uniqueness condition is provided on the basis of a suitable variational formulation.
\end{abstract}

\section{Introduction}

Coupled thermomechanical problem arises in a variety of important fields of application, including casting, metal forming, machining and other manufacturing processes, structural models, and others.

Green and Naghdi (GN) introduced a theory in which heat propagates as thermal waves at finite speed and does not necessarily involve energy dissipation. Another property of the GN theory of type II is the fact that the entropy flux vector is determined by means of the same potential as the mechanical stress tensor. Motivated by the procedure presented in $[1,2]$, this paper is concerned with the formulation of variational principles characterizing the solutions of the coupled thermomechanical problem for the GN model without dissipation, that is, type II. The variational characterization of the thermoelastic problem means the identification of a functional whose stationary points are solutions of the problem. Once this functional is known, the solutions of the problem can be identified with certain extrema of the functional.

Following the pioneering work of Biot [3], the variational forms of the coupled thermoelastic and thermoviscoelastic problems for the classical Fourier model have been investigated in several papers; see, for example, [2,4-9] for the case of thermomechanical coupling in dissipative materials. Moreover consistent variational principles for structural problems concerning elastic and elastoplastic models in isothermal conditions are well developed; see, for example, [10-12].

On the contrary variational formulations for the GN theory of type II are addressed in a few papers; see, for example, $[1,13,14]$.

In this paper the GN thermoelastic coupled structural model without dissipation is formulated in a suitable form so that we can provide the methodology to build the complete family of all the admissible variational formulations associated with the considered GN model. It is well-known that the GN theory of type II has been developed as a dynamic theory, but an important prerequisite for its use is a thorough understanding of the corresponding problems in which the dynamical effects are disregarded; see, for example, [15].

An advantage of such an approach consists in the fact that the boundary-value problem for the thermoelastic model is formulated in such a way that the model can be cast in terms of a multivalued structural operator defined in terms of all the state variables. This operator encompasses in a unique expression the field equation, the constitutive relations, the constraint relations and the initial conditions which describe the considered thermoelastic structural model.

The related non-smooth potential can then be evaluated by a direct integration along a ray in the operator domain and depends on all the state variables involved in the model. Appraising the generalized gradient of the non-smooth potential [10] and imposing its stationarity, the operator formulation of the problem is recovered. 
Hence a general procedure to derive variational formulations within the incremental framework for the considered GN coupled thermoelastic model without dissipation is formulated. It is then shown how a family of mixed variational formulations, associated with the considered GN model, can be obtained following a direct and general procedure by enforcing the fulfilment of field equations and constraint conditions.

The possibility to formulate the coupled GN thermoelastic problem in a variational form has a number of consequences and some beneficial effects. For instance, the variational framework allows one to apply the tools of the calculus of variations to the analysis of the solutions of the problem. In particular, conditions for the existence (see, e.g., $[16,17])$ and uniqueness of the solution are based on the variational framework.

Accordingly the condition for the uniqueness of the solution of the considered model is provided by means of a minimization principle.

\section{Thermoelastic Structural Problem}

Throughout the paper bold-face letters are associated with vectors and tensors. The scalar product between dual quantities (simple or double index saturation operation between vectors or tensors) is denoted by $*$. A superimposed dot means differentiation with respect to time and the symbol $\nabla$ denotes the gradient operator.

In small strain analysis the theory of thermoelasticity without energy dissipation, as described in Green and Naghdi [18], is considered. Such a theory is based on the introduction of a scalar thermal displacement $\alpha$ defined as

$$
\alpha(\mathbf{x}, t)=\int_{0}^{t} \vartheta(\mathbf{x}, \tau) d \tau+\alpha_{o}(\mathbf{x}, 0),
$$

where $\mathbf{x}$ is a point pertaining to a thermoelastic body defined on a regular bounded domain $\Omega$ of an Euclidean space, $\vartheta=$ $\theta-\theta_{r}$ represents the temperature variation from the uniform reference temperature $\theta_{r}$, and $\alpha_{o}(\mathbf{x}, 0)$ is the initial value of the thermal displacement at the time $t=0$. Accordingly the time derivative of $\alpha$ is the temperature variation, that is, $\dot{\alpha}=\theta-\theta_{r}$.

The mechanical and thermal parts of the thermoelastic model are hereafter defined.

Let $\mathscr{D}$ denote the linear space of strain tensors $\varepsilon(\mathbf{x}, t)$ and $\mathcal{S}$ denote the dual space of stress tensors $\sigma(\mathbf{x}, t)$. The inner product in the dual spaces $\langle\cdot, \cdot\rangle$ has the mechanical meaning of the internal virtual work, that is

$$
\langle\sigma, \varepsilon\rangle=\int_{\Omega} \boldsymbol{\sigma}(\mathbf{x}, t) * \varepsilon(\mathbf{x}, t) d \mathbf{x} .
$$

The linear space of displacements $\mathcal{U}$ is denoted by $\mathbf{u}$. The linear space of forces is $\mathscr{F}$ and is placed in separating duality with $\mathcal{U}$ by a nondegenerate bilinear form $\langle\cdot, \cdot\rangle$ which has the physical meaning of external virtual work. For avoiding proliferation of symbols, the internal and external virtual works are denoted by the same symbol. Conforming displacement fields satisfy homogeneous boundary conditions and belong to a closed linear subspace $\mathscr{L} \subset \mathscr{U}$.
The kinematic operator $\mathbf{B}$ is a bounded linear operator from $\mathcal{U}$ to the space of square integrable strain fields $\varepsilon \in \mathscr{D}$. The subspace of external forces $\mathscr{F}$ is the dual space of $\mathscr{U}$. The equilibrium operator is the continuous operator $\mathbf{B}^{\prime}$ from $\mathcal{S}$ to $\mathscr{F}$ which is dual of $\mathbf{B}$. Let $\ell=\{\mathbf{t}, \mathbf{b}\} \in \mathscr{F}$ be the load functional where $\mathbf{t}$ and $\mathbf{b}$ denote the tractions and the body forces [12, 19].

The equilibrium equation and the compatibility condition are given by

$$
\ell+\mathbf{r}=\mathbf{B}^{\prime} \sigma, \quad \varepsilon=\mathbf{B u}
$$

where $\ell, \mathbf{r} \in \mathscr{F}, \sigma \in \mathcal{S}$ and $\varepsilon \in \mathscr{D}, \mathbf{u} \in \mathcal{U}$.

The external relation between reactions and displacements is provided by

$$
\mathbf{r} \in \partial \Upsilon(\mathbf{u})
$$

being $\Upsilon$ a concave function, and the symbol $\partial$ denotes the sub(super)differential of convex (concave) functions [20]. Accordingly, the inverse relation is expressed as

$$
\mathbf{u} \in \partial \Upsilon^{*}(\mathbf{r})
$$

where the concave function $\Upsilon^{*}$ represents the conjugate of $\Upsilon$ and the Fenchel's relation holds

$$
\Upsilon(\mathbf{u})+\Upsilon^{*}(\mathbf{r})=\langle\mathbf{r}, \mathbf{u}\rangle .
$$

Different expressions can be given to the functional $\Upsilon$ depending on the type of external constraints such as bilateral, unilateral, elastic, or convex. For future reference the expressions of $\Upsilon$ and $\Upsilon^{*}$ are specialized to the case of external frictionless bilateral constraints with homogeneous boundary conditions. Noting that the subspace of the external constraint reactions $\mathscr{R}$ is the orthogonal complement of the subspace of conforming displacements $\mathscr{L}$, that is $\mathscr{R}=\mathscr{L}^{\perp}$, the functional $\Upsilon$ turns out to be

$$
\Upsilon(\mathbf{u})=\prod_{\mathscr{L}}(\mathbf{u})= \begin{cases}0 & \text { if } \mathbf{u} \in \mathscr{L} \\ -\infty & \text { otherwise }\end{cases}
$$

and a direct evaluation shows that its conjugate $\Upsilon^{*}$ is given by:

$$
\Upsilon^{*}(\mathbf{r})=\prod_{\mathscr{L}^{\perp}}(\mathbf{r})= \begin{cases}0 & \text { if } \mathbf{r} \in \mathscr{L}^{\perp} \\ -\infty & \text { otherwise }\end{cases}
$$

The proposed framework has the advantage that the formulation of the thermal model is similar to the mechanical one, and the thermoelastic problem turns out to be suitable to build a general variational formulation as shown in the next section.

The linear space of thermal displacement $\alpha$ is $\mathscr{A}$. The rate of heat flow into the body by heat sources $r$ and the boundary heat fluxes $\bar{q}$ belong to the space $\mathscr{H} \subset \mathscr{A}^{\prime}$, dual of $\mathscr{A}$, of square integrable fields on $\Omega$. The external thermal forces are collected in the set $\ell_{\theta}=\left\{\bar{q},-r / \theta_{r}\right\} \in \mathscr{A}^{\prime}$. 
The kinematic thermal operator $\mathbf{G} \in \operatorname{Lin}\{\mathscr{A}, \mathscr{C}\}$ is a bounded linear operator, and a thermal gradient $\mathbf{g} \in \mathscr{G}$ is said to be thermally compatible if there exists an admissible thermal displacement field $\alpha$ such that $\mathbf{g}=\mathbf{G} \alpha$. The thermal balance equation is given by $\mathbf{G}^{\prime} \mathbf{p}=\dot{\eta}+\ell_{\theta}+\gamma$, where $\mathbf{G}^{\prime}$ is the dual operator of $\mathbf{G}$ and $\mathbf{p}$ is the entropy flux vector.

Constraint conditions can be fit in field equations by noting that the external relation between reactive thermal forces $\gamma$ and thermal displacements $\alpha$ is provided by the equivalent relations:

$$
\gamma \in \partial \Pi(\alpha) \Longleftrightarrow \alpha \in \partial \Pi^{*}(\gamma) \Longleftrightarrow \Pi(\alpha)+\Pi^{*}(\gamma)=\langle\gamma, \alpha\rangle,
$$

being $\Pi$ and $\Pi^{*}$ conjugate convex functionals. The equality $(9)_{3}$ represents the Fenchel's relation.

Homogeneous constraints on thermal displacement fields are formulated by considering that thermal displacement fields belong to the subspace $\mathscr{K}$ and are said to be admissible. Reactive thermal forces belong to the orthogonal complement $\mathscr{K}^{\perp}$ of $\mathscr{K}$. Then the functional $\Pi$ is the indicator of $\mathscr{K}$ :

$$
\Pi(\alpha)=\coprod_{\mathscr{K}}(\alpha)= \begin{cases}0 & \text { if } \alpha \in \mathscr{K} \\ +\infty & \text { otherwise }\end{cases}
$$

and its conjugate $\Pi^{*}$ is given by

$$
\Pi^{*}(\gamma)=\coprod_{\mathscr{K}^{\perp}}(\gamma)= \begin{cases}0 & \text { if } \gamma \in \mathscr{K}^{\perp} \\ +\infty & \text { otherwise. }\end{cases}
$$

Accordingly the relations governing the quasistatic thermoelastic structural problem without energy dissipation for given mechanical and thermal loads $\ell$ and $\ell_{\vartheta}$ in the time interval $I=[0, t]$ can be collected in the following form:

$$
\begin{aligned}
& \text { equilibrium: } \mathbf{B}^{\prime} \sigma=\ell+\mathbf{r}, \\
& \text { thermal balance equation: } \mathbf{G}^{\prime} \mathbf{p}=\dot{\eta}+\ell_{\theta}+\gamma \\
& \text { compatibility: } \mathbf{B u}=\varepsilon \\
& \text { thermal compatibility: } \mathbf{G} \alpha=\mathbf{g}, \\
& \text { constitutive relation: } \sigma=d_{\varepsilon} \Phi(\varepsilon, \eta) \\
& \text { constitutive relation: } \dot{\alpha}=d_{\eta} \Phi(\varepsilon, \eta) \\
& \text { constitutive relation: } \mathbf{p}=-d \Psi(\mathbf{g}) \\
& \text { external relation: } \mathbf{u} \in \partial \Upsilon^{*}(\mathbf{r}) \\
& \text { thermal external relation: } \alpha \in \partial \Pi^{*}(\gamma)
\end{aligned}
$$

The initial thermal conditions are considered in the following form:

$$
\alpha(\mathbf{x}, 0)=\alpha_{o}(\mathbf{x}), \quad \dot{\alpha}(\mathbf{x}, 0)=\theta_{o}(\mathbf{x}) \quad \text { with } \mathbf{x} \in \Omega,
$$

where $\alpha_{o}$ and $\theta_{o}$ are, respectively, a prescribed initial thermal displacement and temperature in $\Omega$.
The thermoelastic functionals $\Phi$ and $\Psi$ provide the thermoelastic constitutive relations of the considered GN model.

In the case of a linear coupled thermoelastic behaviour the expression of the functional $\Phi$ is

$$
\Phi(\varepsilon, \eta)=\frac{1}{2}\left\langle\mathbb{E}_{s} \varepsilon, \varepsilon\right\rangle-\frac{\theta_{r}}{c_{V}^{r}}\langle\eta \mathbf{M}, \varepsilon\rangle+\frac{\theta_{r}}{2 c_{V}^{r}}\langle\eta, \eta\rangle,
$$

where the first term at the r.h.s. above is the isentropic elastic strain energy and the second one represents the thermoelastic coupling. The parameter $c_{V}^{r}$ is the specific heat at constant strain at the reference state with temperature $\theta_{r}, \mathbb{E}_{s}=\mathbb{E}+$ $\theta_{r}(\mathbf{M} \otimes \mathbf{M}) / c_{V}^{r}$ is the symmetric and positive definite isentropic elastic moduli fourth-order tensor. The second-order thermal expansion tensor $\mathbf{M}$ is self-adjoint, that is, $\mathbf{M}^{\prime}=\mathbf{M}$. Moreover the thermal function $\Psi$ is given by

$$
\Psi(\mathbf{g})=\frac{1}{2 \theta_{r}}\langle\mathbf{K g}, \mathbf{g}\rangle,
$$

where $\mathbf{K}$ is the tensor of conductivity moduli, symmetric and positive definite.

A solution of the thermoelastic structural problem can be achieved by a finite element approach (see e.g., [21, 22]) and can be obtained starting from a suitable mixed variational formulation. Hence the definition of a general variational formulation which allows one to derive variational principles with different combination of the state variables, without ad hoc procedure, plays a central role.

The time integral of the constitutive relation $(12)_{6}$ in the interval $I$ is given by

$$
\alpha-\alpha_{o}=\int_{I} d_{\eta} \Phi(\varepsilon, \eta) d \tau=-\frac{\theta_{r}}{c_{V}^{r}} \int_{I} \mathbf{M} * \varepsilon d \tau+\frac{\theta_{r}}{c_{V}^{r}} \int_{I} \eta d \tau,
$$

where we have set $\alpha_{o}=\alpha_{o}(\mathbf{x}, 0)$.

Moreover the time integral of the thermal balance equation $(12)_{2}$ in the interval $I$ is given by

$$
\int_{I} \mathbf{G}^{\prime} \mathbf{p} d \tau=\int_{I}\left(\ell_{\vartheta}+\gamma\right) d \tau+\eta-\mathbf{M} * \varepsilon_{o}-\frac{c_{V}^{r}}{\theta_{r}} \theta_{o},
$$

where we have set $\varepsilon_{o}=\varepsilon(\mathbf{x}, 0)$ and $\theta_{o}=\theta_{o}(\mathbf{x})$. In fact, the constitutive relation $(12)_{6}$, evaluated at the initial time $t=$ 0 , provides the equality $\dot{\alpha}(\mathbf{x}, 0)=\left.d_{\eta} \Phi(\varepsilon, \eta)\right|_{(\mathbf{x}, 0)}=-\theta_{r} \mathbf{M} *$ $\varepsilon(\mathbf{x}, 0) / c_{V}^{r}+\theta_{r} \eta(\mathbf{x}, 0) / c_{V}^{r}$ which yields the relation $\eta(\mathbf{x}, 0)=$ $\mathbf{M} * \varepsilon(\mathbf{x}, 0)+c_{V}^{r} \theta_{o}(\mathbf{x}) / \theta_{r}$.

Accordingly the thermoelastic structural problem (12) is rewritten in the following form where the initial conditions (13) are incorporated into the field equations (12):

equilibrium: $\mathbf{B}^{\prime} \boldsymbol{\sigma}=\ell+\mathbf{r}$,

compatibility: $\mathbf{B u}=\boldsymbol{\varepsilon}$,

constitutive relation: $\sigma=d_{\varepsilon} \Phi(\varepsilon, \eta)$,

constitutive relation: $\alpha-\alpha_{o}=\int_{I} d_{\eta} \Phi(\varepsilon, \eta) d \tau$,

external relation: $\mathbf{u} \in \partial \Upsilon^{*}(\mathbf{r})$, 
thermal balance equation: $\int_{I} \mathbf{G}^{\prime} \mathbf{p} d \tau=\int_{I}\left(\ell_{\vartheta}+\gamma\right) d \tau+\eta$,

$$
-\mathbf{M} * \varepsilon_{o}-\frac{c_{V}^{r}}{\theta_{r}} \theta_{o},
$$

thermal compatibility: $\mathbf{G} \alpha=\mathbf{g}$,

constitutive relation: $\mathbf{p}=-d \Psi(\mathbf{g})$,

thermal external relation: $\alpha \in \partial \Pi^{*}(\gamma)$.

\section{General Mixed Variational Principle}

Introducing the product space $\mathscr{W}=\mathscr{U} \times \mathcal{S} \times \mathscr{D} \times \mathscr{A}^{\prime} \times \mathscr{F} \times$ $\mathscr{A} \times \mathscr{G}^{\prime} \times \mathscr{G} \times \mathscr{A}^{\prime}$ and its dual space $\mathscr{W}^{\prime}=\mathscr{F} \times \mathscr{D} \times \mathscr{S} \times \mathscr{A} \times$ $\mathscr{U} \times \mathscr{A}^{\prime} \times \mathscr{G} \times \mathscr{G}^{\prime} \times \mathscr{A}$, the thermoelastic structural problem without dissipation (18) can be collected in terms of the global multivalued structural operators $\mathbf{S}$ and $\widehat{\mathbf{S}}: \mathscr{W} \rightarrow$ $\mathscr{W}^{\prime}$ governing the whole problem

$\mathbf{0} \in \int_{I}\left[\mathbf{S}(\mathbf{w})+\mathbf{w}_{o}\right] d t+\widehat{\mathbf{S}}(\mathbf{w})+\widehat{\mathbf{w}}_{o}, \quad \mathbf{w} \in \mathscr{W}, \mathbf{w}_{o}, \widehat{\mathbf{w}}_{o} \in \mathscr{W}^{\prime}$.

The explicit expression of the structural operators $\mathbf{S}, \widehat{\mathbf{S}}$ and of the vectors $\mathbf{w}, \mathbf{w}_{o}, \widehat{\mathbf{w}}_{o}$ are given by:

$$
\begin{aligned}
& \mathbf{S}=\left[\begin{array}{cccccccccc}
\mathbf{0} & \mathbf{B}^{\prime} & \mathbf{0} & 0 & -I_{\mathscr{F}} & 0 & \mathbf{0} & \mathbf{0} & 0 \\
\mathbf{B} & \mathbf{0} & -I_{\mathscr{D}} & 0 & \mathbf{0} & 0 & \mathbf{0} & \mathbf{0} & 0 \\
\mathbf{0} & -I_{\mathcal{S}} & & & & \mathbf{0} & 0 & \mathbf{0} & \mathbf{0} & 0 \\
& & & d \Phi & & & & & \\
0 & 0 & & & \mathbf{0} & 0 & \mathbf{0} & \mathbf{0} & 0 \\
-I_{\mathscr{U}} & \mathbf{0} & \mathbf{0} & & 0 & \partial \Upsilon^{*} & 0 & \mathbf{0} & \mathbf{0} & 0 \\
\mathbf{0} & \mathbf{0} & \mathbf{0} & 0 & \mathbf{0} & 0 & \mathbf{G}^{\prime} & \mathbf{0} & -I_{\mathscr{A}}^{\prime} \\
\mathbf{0} & \mathbf{0} & \mathbf{0} & 0 & \mathbf{0} & \mathbf{G} & \mathbf{0} & -I_{\mathscr{G}} & 0 \\
\mathbf{0} & \mathbf{0} & \mathbf{0} & 0 & \mathbf{0} & 0 & -I_{\mathscr{G}^{\prime}} & -d \Psi & 0 \\
\mathbf{0} & \mathbf{0} & \mathbf{0} & 0 & \mathbf{0} & -I_{\mathscr{A}} & \mathbf{0} & \mathbf{0} & \partial \Pi^{*}
\end{array}\right]
\end{aligned}
$$

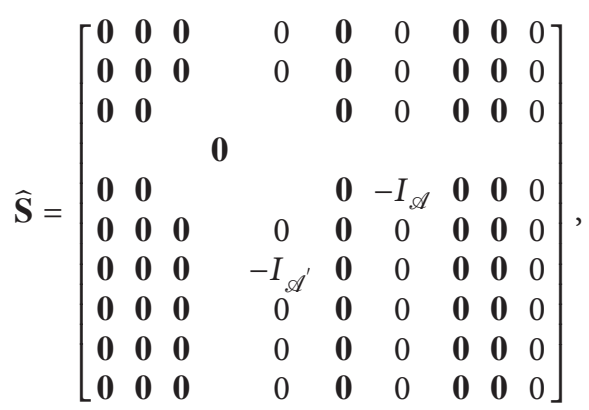

$$
\begin{aligned}
& \mathbf{w}^{T}=\left[\begin{array}{lllllllll}
\mathbf{u} & \boldsymbol{\sigma} & \boldsymbol{\varepsilon} & \eta & \mathbf{r} & \alpha & \mathbf{p} & \mathbf{g} & \gamma
\end{array}\right]^{T}, \\
& \mathbf{w}_{o}^{T}=\left[\begin{array}{lllllllll}
-\ell & \mathbf{0} & \mathbf{0} & 0 & \mathbf{0} & -\ell_{\vartheta} & \mathbf{0} & \mathbf{0} & 0
\end{array}\right]^{T},
\end{aligned}
$$

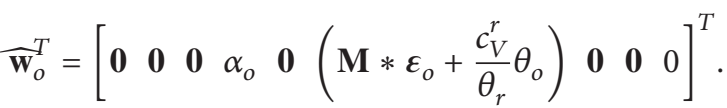

The operators $\mathbf{S}$ and $\widehat{\mathbf{S}}$ turn out to be integrable by virtue of the duality existing between the operators $\mathbf{B}, \mathbf{G}, \mathbf{M}, I_{\mathscr{U}}, I_{\mathscr{D}}$, $I_{\mathscr{A}}, I_{\mathscr{G}}$ and $\mathbf{B}^{\prime}, \mathbf{G}^{\prime}, \mathbf{M}^{\prime}, I_{\mathscr{F}}, I_{\mathscr{S}}, I_{\mathscr{A}^{\prime}}, I_{\mathscr{G}^{\prime}}$, the conservativity of $d \Phi, d \Psi$ and the conservativity of the super(sub)differentials $\partial \Upsilon^{*}$ and $\partial \Pi^{*}$.

The related potential can be evaluated by a direct integration along a straight line in the space $\mathscr{W}$ starting from its origin to get [23]:

$$
\begin{aligned}
& M(\mathbf{u}, \boldsymbol{\sigma}, \boldsymbol{\varepsilon}, \eta, \mathbf{r}, \alpha, \mathbf{p}, \mathbf{g}, \gamma) \\
& =\int_{I}\left[\int_{0}^{1}\langle\mathbf{S}(\zeta \mathbf{w}), \mathbf{w}\rangle d \zeta-\langle\ell, \mathbf{u}\rangle-\left\langle\ell_{\vartheta}, \alpha\right\rangle\right] d \tau \\
& \quad+\int_{0}^{1}\langle\widehat{\mathbf{S}}(\zeta \mathbf{w}), \mathbf{w}\rangle d \zeta+\left\langle\mathbf{M} * \varepsilon_{o}+\frac{c_{V}^{r}}{\theta_{r}} \theta_{o}, \alpha\right\rangle \\
& \quad+\left\langle\eta, \alpha_{o}\right\rangle .
\end{aligned}
$$

Hence it turns out to be

$M(\mathbf{u}, \sigma, \varepsilon, \eta, \mathbf{r}, \alpha, \mathbf{p}, \mathbf{g}, \gamma)$

$$
\begin{gathered}
=\int_{I}\left[\Phi(\varepsilon, \eta)-\Psi(\mathbf{g})+\Upsilon^{*}(\mathbf{r})+\Pi^{*}(\gamma)-\langle\boldsymbol{\sigma}, \varepsilon\rangle+\langle\boldsymbol{\sigma}, \mathbf{B u}\rangle\right. \\
\left.-\langle\ell+\mathbf{r}, \mathbf{u}\rangle-\langle\mathbf{p}, \mathbf{g}\rangle+\langle\mathbf{p}, \mathbf{G} \alpha\rangle-\left\langle\ell_{\vartheta}+\gamma, \alpha\right\rangle\right] d \tau \\
-\left\langle\eta, \alpha-\alpha_{o}\right\rangle+\left\langle\mathbf{M} * \varepsilon_{o}+\frac{c_{V}^{r}}{\theta_{r}} \theta_{o}, \alpha\right\rangle .
\end{gathered}
$$

The potential $M$ turns out to be linear in $(\mathbf{u}, \sigma, \alpha, \mathbf{p})$, jointly convex with respect to the state variables $(\varepsilon, \eta, \gamma)$ and jointly concave with respect to $(\mathbf{g}, \mathbf{r})$. The following statement then holds.

Proposition 1. The set of state variables $(\mathbf{u}, \sigma, \varepsilon, \eta, \mathbf{r}, \alpha, \mathbf{p}, \mathbf{g}$, $\gamma$ ) is a solution of the saddle problem:

$$
\min _{\varepsilon, \eta, \gamma} \max _{\mathbf{g}, \mathbf{r}} \operatorname{stat}_{\mathbf{u}, \sigma, \alpha, \mathbf{p}} M(\mathbf{u}, \boldsymbol{\sigma}, \varepsilon, \eta, \mathbf{r}, \alpha, \mathbf{p}, \mathbf{g}, \gamma)
$$

if and only if it is a solution of the thermoelastic structural problem without dissipation (18).

The stationary condition of $M$, enforced at the point $(\mathbf{u}, \sigma$, $\varepsilon, \eta, \mathbf{r}, \alpha, \mathbf{p}, \mathbf{g}, \gamma)$, yields back the thermoelastic structural problem. In fact the stationarity of $M$ is:

$$
(\mathbf{0}, \mathbf{0}, \mathbf{0}, 0, \mathbf{0}, 0, \mathbf{0}, \mathbf{0}, 0) \in \partial M(\mathbf{u}, \boldsymbol{\sigma}, \varepsilon, \eta, \mathbf{r}, \alpha, \mathbf{p}, \mathbf{g}, \gamma)
$$


which can be rewritten in the following form

$$
\begin{aligned}
& \mathbf{0}=d_{\mathbf{u}} M(\mathbf{u}, \boldsymbol{\sigma}, \varepsilon, \eta, \mathbf{r}, \alpha, \mathbf{p}, \mathbf{g}, \gamma), \\
& \mathbf{0}=d_{\sigma} M(\mathbf{u}, \boldsymbol{\sigma}, \varepsilon, \eta, \mathbf{r}, \alpha, \mathbf{p}, \mathbf{g}, \gamma), \\
& \mathbf{0}=d_{\varepsilon} M(\mathbf{u}, \boldsymbol{\sigma}, \varepsilon, \eta, \mathbf{r}, \alpha, \mathbf{p}, \mathbf{g}, \gamma), \\
& 0=d_{\eta} M(\mathbf{u}, \boldsymbol{\sigma}, \varepsilon, \eta, \mathbf{r}, \alpha, \mathbf{p}, \mathbf{g}, \gamma), \\
& \mathbf{0} \in \partial_{\mathbf{r}} M(\mathbf{u}, \boldsymbol{\sigma}, \varepsilon, \eta, \mathbf{r}, \alpha, \mathbf{p}, \mathbf{g}, \gamma), \\
& 0=d_{\alpha} M(\mathbf{u}, \boldsymbol{\sigma}, \varepsilon, \eta, \mathbf{r}, \alpha, \mathbf{p}, \mathbf{g}, \gamma), \\
& \mathbf{0}=d_{\mathbf{p}} M(\mathbf{u}, \boldsymbol{\sigma}, \varepsilon, \eta, \mathbf{r}, \alpha, \mathbf{p}, \mathbf{g}, \gamma), \\
& \mathbf{0}=d_{\mathbf{g}} M(\mathbf{u}, \boldsymbol{\sigma}, \varepsilon, \eta, \mathbf{r}, \alpha, \mathbf{p}, \mathbf{g}, \gamma), \\
& 0 \in \partial_{\gamma} M(\mathbf{u}, \boldsymbol{\sigma}, \varepsilon, \eta, \mathbf{r}, \alpha, \mathbf{p}, \mathbf{g}, \gamma) .
\end{aligned}
$$

From a mechanical point of view, the relation $(25)_{1}$ yields the equilibrium equation:

$$
\mathbf{B}^{\prime} \sigma=\ell+\mathbf{r}
$$

the relation $(25)_{2}$ provides the compatibility condition:

$$
\mathbf{B u}=\varepsilon,
$$

the relations $(25)_{3-4}$ yield the constitutive relations:

$$
\sigma=d_{\varepsilon} \Phi(\varepsilon, \eta), \quad \alpha-\alpha_{o}=\int_{I} d_{\eta} \Phi(\varepsilon, \eta) d \tau,
$$

the relation $(25)_{5}$ yields the external constraint conditions:

$$
\mathbf{u} \in \partial \Upsilon^{*}(\mathbf{r})
$$

the relation $(25)_{6}$ yields the thermal balance condition:

$$
\int_{I} \mathbf{G}^{\prime} \mathbf{p} d \tau=\int_{I}\left(\ell_{\vartheta}+\gamma\right) d \tau+\eta-\mathbf{M} * \varepsilon_{o}-\frac{c_{V}^{r}}{\theta_{r}} \theta_{o},
$$

the relation $(25)_{7}$ yields the thermal compatibility:

$$
\mathbf{g}=\mathbf{G} \alpha,
$$

the relation $(25)_{8}$ yields the thermal constitutive relation:

$$
\mathbf{p}=-d \Psi(\mathbf{g}),
$$

and, finally, the relation $(25)_{9}$ yields the thermal external relation:

$$
\alpha \in \partial \Pi^{*}(\gamma)
$$

Hence, performing the super(sub)differentials appearing in (25), the structural problem (18) is recovered.

3.1. Mixed Variational Principles. A family of potentials can be recovered from the potential $M$ by enforcing the fulfilment of field equations and of constitutive relations. All these functionals assume the same value when they are evaluated at a solution point of the GN structural problem.
Hence a variational principle in which the external reactions do not appear as independent state variables can be obtained by imposing, in the expression (22) of the potential $M$, the external relations (18) $)_{5,9}$ in terms of Fenchel's equalities (6) and $(9)_{3}$. Hence it turns out to be

$$
\begin{aligned}
& M_{1}(\mathbf{u}, \boldsymbol{\sigma}, \boldsymbol{\varepsilon}, \eta, \alpha, \mathbf{p}, \mathbf{g}) \\
& =\int_{I}[\Phi(\varepsilon, \eta)-\Psi(\mathbf{g})-\Upsilon(\mathbf{u})-\Pi(\alpha)-\langle\boldsymbol{\sigma}, \varepsilon\rangle+\langle\boldsymbol{\sigma}, \mathbf{B u}\rangle \\
& \left.\quad-\langle\ell, \mathbf{u}\rangle-\langle\mathbf{p}, \mathbf{g}\rangle+\langle\mathbf{p}, \mathbf{G} \alpha\rangle-\left\langle\ell_{\vartheta}, \alpha\right\rangle\right] d \tau \\
& \quad-\left\langle\eta, \alpha-\alpha_{o}\right\rangle+\left\langle\mathbf{M} * \varepsilon_{o}+\frac{c_{V}^{r}}{\theta_{r}} \theta_{o}, \alpha\right\rangle
\end{aligned}
$$

and the following statement holds.

Proposition 2. The set of state variables $(\mathbf{u}, \sigma, \varepsilon, \eta, \alpha, \mathbf{p}, \mathbf{g})$ is a solution of the saddle problem:

$$
\min _{\mathbf{u}, \varepsilon, \eta} \max _{\alpha, \mathbf{g}} \underset{\sigma, \mathbf{p}}{\operatorname{stat}} M_{1}(\mathbf{u}, \boldsymbol{\sigma}, \boldsymbol{\varepsilon}, \eta, \alpha, \mathbf{p}, \mathbf{g})
$$

if and only if it is a solution of the thermoelastic structural problem without dissipation (18).

The proof of the mixed GN thermoelastic variational formulation reported in the Proposition 2 is provided in the Appendix. The proofs of the following variational formulations are omitted since they are obtained following a similar reasoning.

Imposing in the expression (34) of the potential $M_{1}$ the compatibility condition $(18)_{2}$ follows as

$$
\begin{aligned}
M_{2}(\mathbf{u}, \eta, \alpha, \mathbf{p}, \mathbf{g})=\int_{I}[ & {[(\mathbf{B u}, \eta)-\Psi(\mathbf{g})-\Upsilon(\mathbf{u})-\Pi(\alpha)} \\
& \left.-\langle\ell, \mathbf{u}\rangle-\langle\mathbf{p}, \mathbf{g}\rangle+\langle\mathbf{p}, \mathbf{G} \alpha\rangle-\left\langle\ell_{\vartheta}, \alpha\right\rangle\right] d \tau \\
& -\left\langle\eta, \alpha-\alpha_{o}\right\rangle+\left\langle\mathbf{M} * \varepsilon_{o}+\frac{c_{V}^{r}}{\theta_{r}} \theta_{o}, \alpha\right\rangle,
\end{aligned}
$$

and the following five-field mixed variational principle holds.

Proposition 3. The set of state variables $(\mathbf{u}, \eta, \alpha, \mathbf{p}, \mathbf{g})$ is a solution of the saddle problem:

$$
\min _{\mathbf{u}, \eta} \max _{\alpha, \mathbf{g}} \underset{\mathbf{p}}{\operatorname{stat}} M_{2}(\mathbf{u}, \eta, \alpha, \mathbf{p}, \mathbf{g})
$$

if and only if it is a solution of the thermoelastic structural problem without dissipation (18). 
Imposing in the expression (36) of the potential $M_{2}$ the thermal compatibility condition $(18)_{7}$ follows:

$$
\begin{aligned}
& M_{3}(\mathbf{u}, \eta, \alpha) \\
& =\int_{I}[\Phi(\mathbf{B u}, \eta)-\Psi(\mathbf{G} \alpha)-\Upsilon(\mathbf{u}) \\
& \left.\quad-\Pi(\alpha)-\langle\ell, \mathbf{u}\rangle-\left\langle\ell_{\vartheta}, \alpha\right\rangle\right] d \tau \\
& \quad-\left\langle\eta, \alpha-\alpha_{o}\right\rangle+\left\langle\mathbf{M} * \varepsilon_{o}+\frac{c_{V}^{r}}{\theta_{r}} \theta_{o}, \alpha\right\rangle,
\end{aligned}
$$

and the following three-field mixed variational formulation holds.

Proposition 4. The set of state variables $(\mathbf{u}, \eta, \alpha)$ is a solution of the saddle problem:

$$
\min _{\mathbf{u}, \eta} \max _{\alpha} M_{3}(\mathbf{u}, \eta, \alpha)
$$

if and only if it is a solution of the thermoelastic structural problem without dissipation (18).

From a mechanical point of view, the specialization of the potential $M_{3}$ to the Cauchy model for a linear coupled thermoelastic behaviour, external frictionless bilateral constrains, and homogeneous thermal boundary conditions is provided. The functionals $\Phi$ and $\Psi$, see (14) and (15), are

$\Phi(\varepsilon, \eta)=\frac{1}{2} \int_{\Omega} \mathbb{E}_{s} \varepsilon * \varepsilon d \mathbf{x}-\frac{\theta_{r}}{c_{V}^{r}} \int_{\Omega} \eta \mathbf{M} * \varepsilon d \mathbf{x}+\frac{\theta_{r}}{2 c_{V}^{r}} \int_{\Omega} \eta^{2} d \mathbf{x}$,

$\Psi(\mathbf{g})=\frac{1}{2 \theta_{r}} \int_{\Omega} \mathbf{K g} * \mathbf{g} d \mathbf{x}$.

Hence the potential $M_{3}$ becomes

$$
\begin{aligned}
M_{3}(\mathbf{u}, \eta, \alpha)= & \int_{I}\left[H^{u}(\mathbf{u})+H^{\text {th }}(\eta, \alpha)\right] d t \\
& -H_{1}^{\text {th }}(\eta, \alpha)-\int_{I} H_{1}^{\text {coup }}(\mathbf{u}, \eta) d t+H_{2}^{\text {coup }}(\alpha),
\end{aligned}
$$

under the condition that the displacement and the thermal displacement are conforming and admissible, that is, $\mathbf{u} \in \mathscr{L}$ and $\alpha \in \mathscr{K}$, where

$$
H^{u}(\mathbf{u})=\frac{1}{2} \int_{\Omega} \mathbb{E}_{s} \mathbf{B u} * \mathbf{B u} d \mathbf{x}-\int_{\Omega} \mathbf{b} * \mathbf{u} d \mathbf{x}-\int_{\partial \Omega} \mathbf{t} * \Gamma \mathbf{u} d \mathbf{x},
$$

is the total potential energy in classical elasticity in terms of the isentropic elastic stiffness, the functionals:

$$
\begin{aligned}
& H^{\text {th }}(\eta, \alpha)=-\frac{1}{2 \theta_{r}} \int_{\Omega} \mathbf{K} \nabla \alpha * \nabla \alpha d \mathbf{x}+\frac{\theta_{r}}{2 c_{V}^{r}} \int_{\Omega} \eta^{2} d \mathbf{x} \\
& \quad+\frac{1}{\theta_{r}} \int_{\Omega} r * \alpha d \mathbf{x}-\int_{\partial \Omega} \bar{q} * \Gamma \alpha d \mathbf{x}, \\
& H_{1}^{\text {th }}(\eta, \alpha)=\int_{\Omega} \eta *\left(\alpha-\alpha_{o}\right) d \mathbf{x},
\end{aligned}
$$

are the mixed thermal part of the potential, and the functionals:

$$
\begin{aligned}
& H_{1}^{\text {coup }}(\mathbf{u}, \eta)=\frac{\theta_{r}}{c_{V}^{r}} \int_{\Omega} \eta \mathbf{M} * \mathbf{B u} d \mathbf{x}, \\
& H_{2}^{\text {coup }}(\alpha)=\int_{\Omega}\left(\mathbf{M} * \varepsilon_{o}+\frac{c_{V}^{r}}{\theta_{r}} \theta_{o}\right) \alpha d \mathbf{x},
\end{aligned}
$$

take into account the thermoelastic coupling.

Minimum principles in structural mechanics are relevant to investigate since solution techniques can be exploited, and existence and uniqueness results can be provided by recourse to functional analysis. Actually, uniqueness of the solution is ensured if the functional to be minimized is strictly convex.

Hence it is important to derive a variational formulation in terms of a minimization problem as hereafter reported.

To this end let us note that the constitutive relation $(18)_{8}$ can be equivalently expressed in terms of the conjugate $\Psi^{*}$ of the thermoelastic functional $\Psi$ in the following form:

$$
\mathbf{g}=d \Psi^{*}(-\mathbf{p})
$$

and the Fenchel's relation hold:

$$
\Psi(\mathbf{g})+\Psi^{*}(-\mathbf{p})=-\langle\mathbf{p}, \mathbf{g}\rangle .
$$

In the case of the Cauchy model with a linear coupled thermoelastic behaviour, the potential $\Psi^{*}$ can be evaluated as the conjugate of (15) and is given by

$$
\Psi^{*}(\mathbf{p})=\frac{\theta_{r}}{2} \int_{\Omega} \mathbf{p} * \mathbf{K}^{-1} \mathbf{p} d \mathbf{x}
$$

Enforcing in the expression of the functional $M$ the thermal constitutive relation $(18)_{8}$ in terms of the Fenchel's equality (46) and the external relation $(18)_{5}$ in terms of Fenchel's equality (6), it turns out to be

$$
\begin{aligned}
& M_{4}(\mathbf{u}, \boldsymbol{\sigma}, \boldsymbol{\varepsilon}, \eta, \alpha, \mathbf{p}, \gamma) \\
& =\int_{I}\left[\Phi(\varepsilon, \eta)+\Psi^{*}(-\mathbf{p})-\Upsilon(\mathbf{u})+\Pi^{*}(\gamma)-\langle\boldsymbol{\sigma}, \boldsymbol{\varepsilon}\rangle\right. \\
& \left.\quad+\langle\boldsymbol{\sigma}, \mathbf{B u}\rangle-\langle\ell, \mathbf{u}\rangle+\langle\mathbf{p}, \mathbf{G} \alpha\rangle-\left\langle\ell_{\vartheta}+\gamma, \alpha\right\rangle\right] d \tau \\
& \quad-\left\langle\eta, \alpha-\alpha_{o}\right\rangle+\left\langle\mathbf{M} * \varepsilon_{o}+\frac{c_{V}^{r}}{\theta_{r}} \theta_{o}, \alpha\right\rangle,
\end{aligned}
$$

and the following minimum principle holds.

Proposition 5. The set of state variables $(\mathbf{u}, \boldsymbol{\sigma}, \boldsymbol{\varepsilon}, \eta, \alpha, \mathbf{p}, \gamma)$ is a solution of the minimum problem:

$$
\min _{(\mathbf{u}, \sigma, \varepsilon, \eta, \alpha, \mathbf{p}, \gamma)} M_{4}(\mathbf{u}, \boldsymbol{\sigma}, \boldsymbol{\varepsilon}, \eta, \alpha, \mathbf{p}, \gamma)
$$

if and only if it is a solution of the thermoelastic structural problem without dissipation (18).

Accordingly, if the thermoelastic functional $\Psi^{*}$ pertaining to the GN constitutive model is strictly convex, the 
functional $M_{4}$ turns out to be strictly convex, and the GN thermoelastic structural model (18) admits a unique solution (if any).

It is worthnoting that, in the linear coupled thermoelastic case, the expression of the functional $\Psi^{*}$ is given by (47) so that it turns out to be a strictly convex functional. Therefore the GN thermoelastic structural model (18) admits a unique solution. The question of existence of the solution is still an challenge problem; see, for example, [17].

\section{Closure}

A variational framework for a class of GN coupled thermomechanical boundary-value problem is presented. The thermoelastic structural model is addressed, and the related general mixed thermoelastic variational formulation in the complete set of state variables is derived starting from the structural model. An advantage of the proposed methodology is that it can be applied to a wide range of structural models, and variational formulations can be obtained following a general reasoning. As a consequence, a family of mixed thermoelastic variational formulations in a reduced number of variables is then contributed. Finally, by appealing to a minimum principle, the connection between uniqueness of the solution and convexity is investigated.

\section{Appendix}

The stationary condition of $M_{1}$, see (34), enforced at the point $(\mathbf{u}, \sigma, \varepsilon, \eta, \alpha, \mathbf{p}, \mathbf{g})$, yields back the thermoelastic structural problem. In fact the stationarity of $M_{1}$ is

$$
(\mathbf{0}, \mathbf{0}, \mathbf{0}, 0,0, \mathbf{0}, \mathbf{0}) \in \partial M_{1}(\mathbf{u}, \sigma, \varepsilon, \eta, \alpha, \mathbf{p}, \mathbf{g}),
$$

which can be rewritten in the following form:

$$
\begin{aligned}
& \mathbf{0} \in \partial_{\mathbf{u}} M_{1}(\mathbf{u}, \boldsymbol{\sigma}, \varepsilon, \eta, \alpha, \mathbf{p}, \mathbf{g}), \\
& \mathbf{0}=d_{\sigma} M_{1}(\mathbf{u}, \boldsymbol{\sigma}, \varepsilon, \eta, \alpha, \mathbf{p}, \mathbf{g}), \\
& \mathbf{0}=d_{\varepsilon} M_{1}(\mathbf{u}, \boldsymbol{\sigma}, \varepsilon, \eta, \alpha, \mathbf{p}, \mathbf{g}), \\
& 0=d_{\eta} M_{1}(\mathbf{u}, \boldsymbol{\sigma}, \varepsilon, \eta, \alpha, \mathbf{p}, \mathbf{g}), \\
& 0 \in \partial_{\alpha} M_{1}(\mathbf{u}, \boldsymbol{\sigma}, \varepsilon, \eta, \alpha, \mathbf{p}, \mathbf{g}), \\
& \mathbf{0}=d_{\mathbf{p}} M_{1}(\mathbf{u}, \boldsymbol{\sigma}, \varepsilon, \eta, \alpha, \mathbf{p}, \mathbf{g}), \\
& \mathbf{0}=d_{\mathbf{g}} M_{1}(\mathbf{u}, \sigma, \varepsilon, \eta, \alpha, \mathbf{p}, \mathbf{g}) .
\end{aligned}
$$

The mechanical interpretation of the relation (A.2) yields the equilibrium equation and the external constraint conditions. In fact there exists a reaction $\mathbf{r} \in \partial \Upsilon(\mathbf{u})$ such that

$$
\mathbf{B}^{\prime} \sigma-\ell=\mathbf{r} .
$$

The relation (A.2) 2 provides the compatibility condition:

$$
\mathbf{B u}=\varepsilon,
$$

the relations $(\text { A.2 })_{3-4}$ yield the constitutive relations:

$$
\sigma=d_{\varepsilon} \Phi(\varepsilon, \eta), \quad \alpha-\alpha_{o}=\int_{I} d_{\eta} \Phi(\varepsilon, \eta) d \tau
$$

the relation (A.2) $)_{5}$ yields the thermal balance condition and the thermal external relations. In fact there exists a thermal reaction $\gamma \in \partial \Pi(\alpha)$ such that

$$
\int_{I} \mathbf{G}^{\prime} \mathbf{p} d \tau-\int_{I}\left(\ell_{\vartheta}+\gamma\right) d \tau-\eta+\mathbf{M} * \varepsilon_{o}+\frac{c_{V}^{r}}{\theta_{r}} \theta_{o}=0
$$

the relation (A.2) 6 yields the thermal compatibility:

$$
\mathbf{g}=\mathbf{G} \alpha,
$$

and the relation $(\mathrm{A} .2)_{7}$ yields the thermal constitutive relation:

$$
\mathbf{p}=-d \Psi(\mathbf{g})
$$

Hence the structural problem (18) is thus recovered.

\section{Acknowledgments}

Research support by “Ministero dell' Istruzione, dell'Università e della Ricerca" of Italy is kindly acknowledged.

\section{References}

[1] S. Bargmann and P. Steinmann, "An incremental variational formulation of dissipative and non-dissipative coupled thermoelasticity for solids," Heat and Mass Transfer, vol. 45, no. 1, pp. 107-116, 2008.

[2] Q. Yang, L. Stainier, and M. Ortiz, "A variational formulation of the coupled thermo-mechanical boundary-value problem for general dissipative solids," Journal of the Mechanics and Physics of Solids, vol. 54, no. 2, pp. 401-424, 2006.

[3] M. A. Biot, "Thermoelasticity and irreversible thermodynamics," Journal of Applied Physics, vol. 27, no. 3, pp. 240-253, 1956.

[4] M. Ben-Amoz, "On a variational theorem in coupled thermoelasticity," Journal of Applied Mechanics, vol. 32, no. 4, pp. 943-945, 1965.

[5] G. Batra, "On a principle of virtual work for thermo-elastic bodies," Journal of Elasticity, vol. 21, no. 2, pp. 131-146, 1989.

[6] G. Herrmann, "On variational principles in thermoelasticity and heat conduction," Quarterly of Applied Mathematics, vol. 22, pp. 151-155, 1963.

[7] G. Lebon, "Variational principles in thermomechanics," in Recent Developments in Thermomechanics of Solids, G. Lebon and P. Perzina, Eds., CISM Courses and Lectures, no. 262, pp. 221-396, Springer, Wien, Austria, 1980.

[8] J. T. Oden and J. N. Reddy, Methods in Theoretical Mechanics, Springer, Berlin, Germany, 1976.

[9] F. Armero and J. C. Simo, "A priori stability estimates and unconditionally stable product formula algorithms for nonlinear coupled thermoplasticity," International Journal of Plasticity, vol. 9, no. 6, pp. 749-782, 1993. 
[10] F. Marotti de Sciarra, "Novel variational formulations for nonlocal plasticity," International Journal of Plasticity, vol. 25, no. 2, pp. 302-331, 2009.

[11] F. Marotti de Sciarra, "A finite element for nonlocal elastic analysis," in Proceedings of the 4th International Conference on Computational Methods for Coupled Problems in Science and Engineering, Kos, Greece, 2011.

[12] F. Marotti de Sciarra, "Hardening plasticity with nonlocal strain damage," International Journal of Plasticity, vol. 34, pp. 114-138, 2012.

[13] D. S. Chandrasekharaiah, "Variational and reciprocal principles in thermoelasticity without energy dissipation," Proceedings of the Indian Academy of Sciences: Mathematical Sciences, vol. 108, no. 2, pp. 209-215, 1998.

[14] S. Chirița and M. Ciarletta, "Reciprocal and variational principles in linear thermoelasticity without energy dissipation," Mechanics Research Communications, vol. 37, no. 3, pp. 271-275, 2010.

[15] R. Quintanilla and J. Sivaloganathan, "Aspects of the nonlinear theory of type II thermoelastostatics," European Journal of Mechanics A, vol. 32, pp. 109-117, 2012.

[16] G. Dal Maso, G. A. Francfort, and R. Toader, "Quasistatic crack growth in nonlinear elasticity," Archive for Rational Mechanics and Analysis, vol. 176, no. 2, pp. 165-225, 2005.

[17] R. Quintanilla, "Existence in thermoelasticity without energy dissipation," Journal of Thermal Stresses, vol. 25, no. 2, pp. 195-202, 2002.

[18] A. E. Green and P. M. Naghdi, "Thermoelasticity without energy dissipation," Journal of Elasticity, vol. 31, no. 3, pp. 189-208, 1993.

[19] R. E. Showalter, Monotone Operators in Banach Space and Nonlinear Partial Differential Equations, American Mathematical Society, Providence, RI, USA, 1997.

[20] R. T. Rockafellar, Convex Analysis, Princeton University Press, Princeton, NJ, USA, 1970.

[21] E. Ruocco and M. Fraldi, "Critical behavior of flat and stiffened shell structures through different kinematical models: a comparative investigation," Thin-Walled Structures, vol. 60, pp. 205-215, 2012.

[22] E. Ruocco and M. Fraldi, "An analytical model for the buckling of plates under mixed boundary conditions," Engineering Structures, vol. 38, pp. 78-88, 2012.

[23] G. Romano, L. Rosati, F. Marotti de Sciarra, and P. Bisegna, "A potential theory for monotone multi-valued operators," Quarterly of Applied Mathematics, vol. 4, no. 4, pp. 613-631, 1993. 

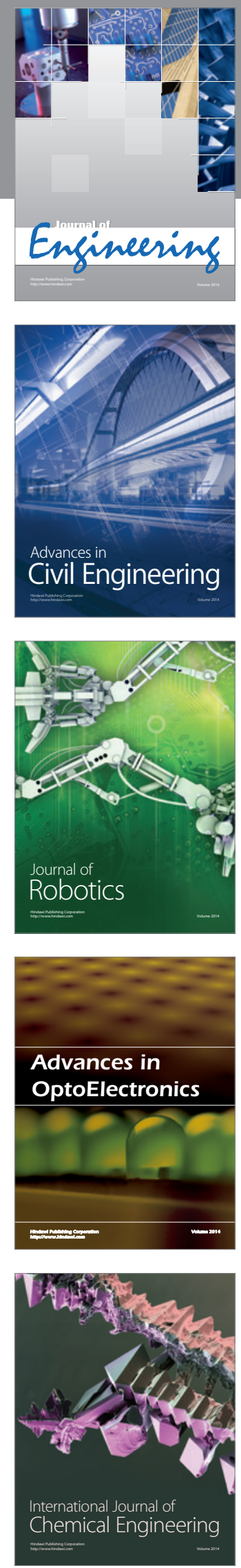

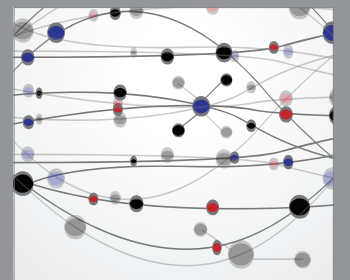

The Scientific World Journal
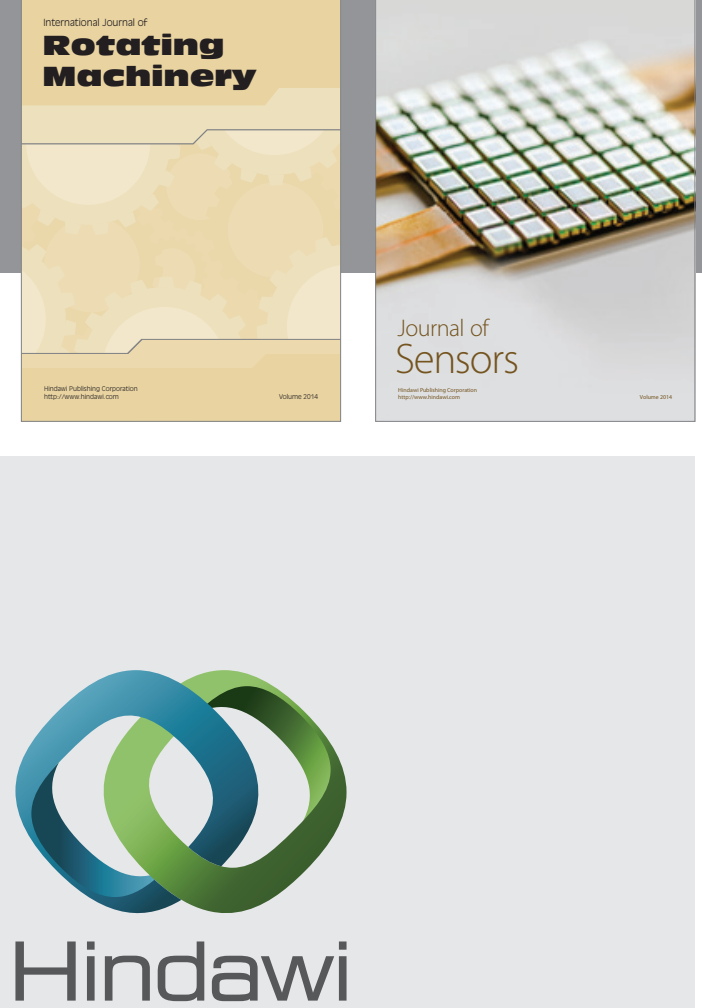

Submit your manuscripts at http://www.hindawi.com
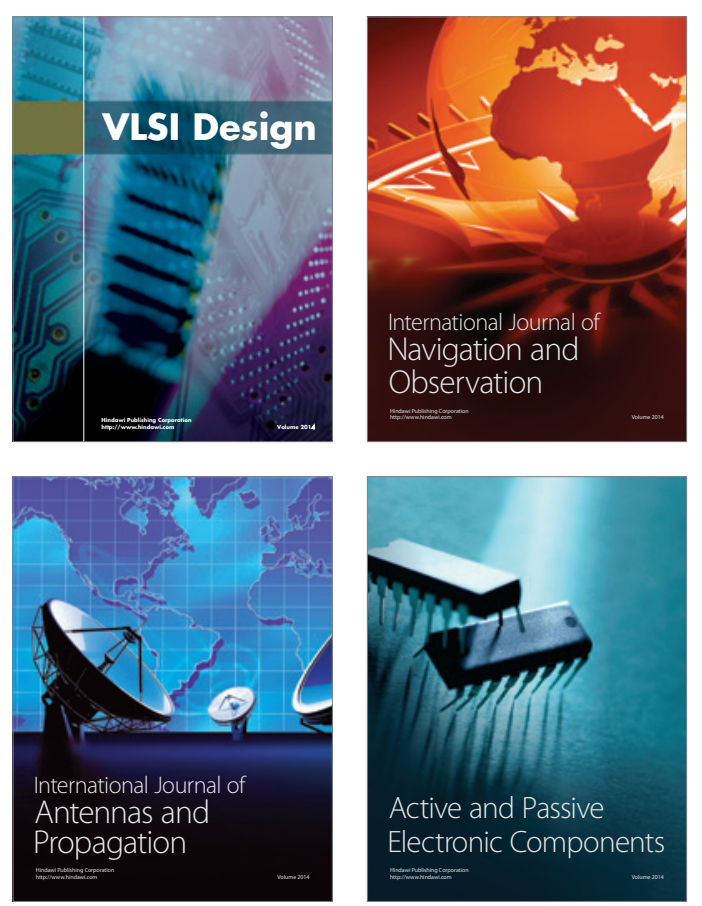
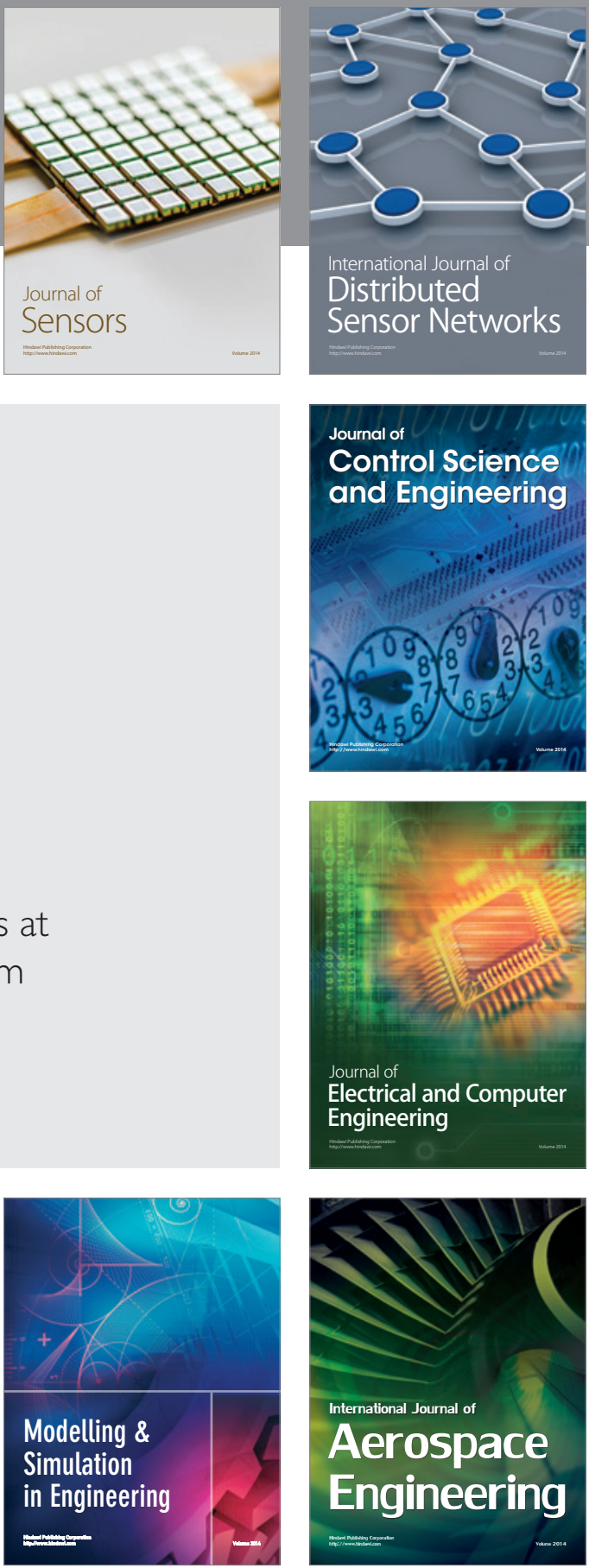

Journal of

Control Science

and Engineering
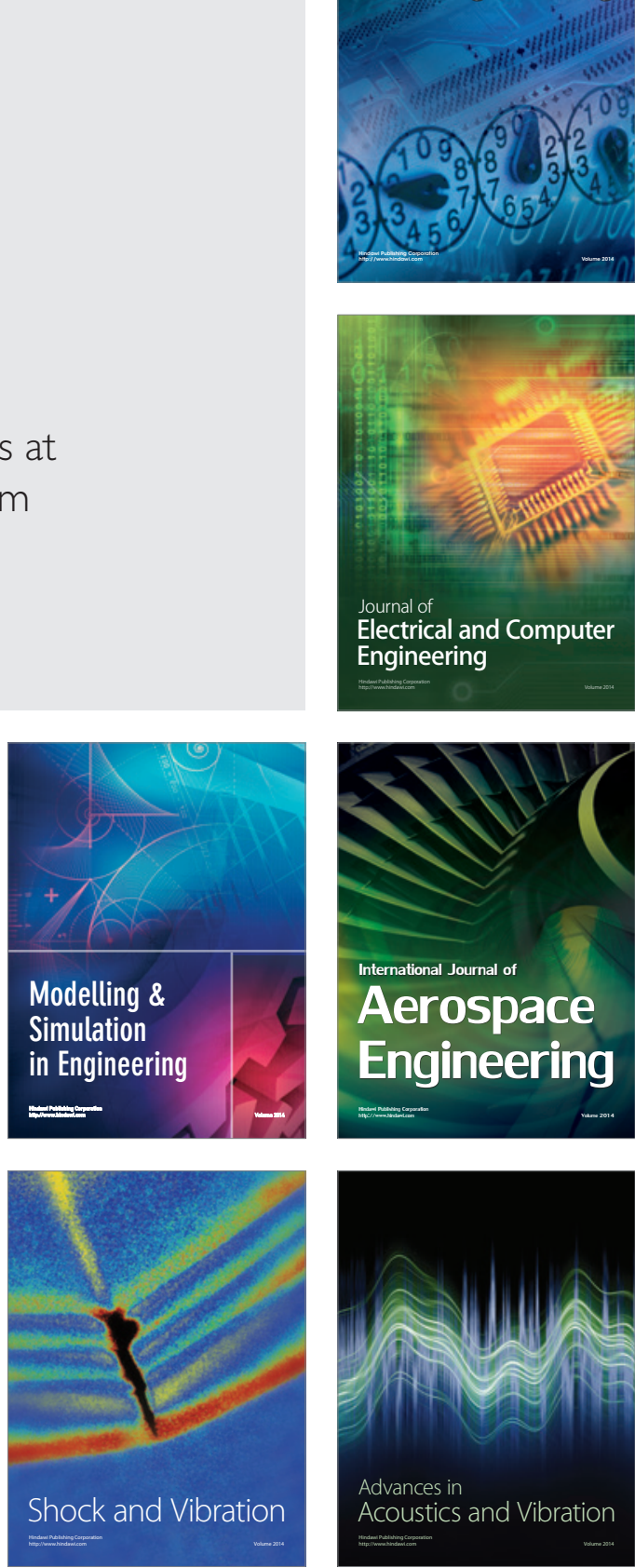